\title{
Corrigendum: Genome-wide adaptive complexes to underground stresses in blind mole rats Spalax
}

Xiaodong Fang, Eviatar Nevo, Lijuan Han, Erez Y. Levanon, Jing Zhao, Aaron Avivi, Denis Larkin, Xuanting Jiang, Sergey Feranchuk, Yabing Zhu, Alla Fishman, Yue Feng, Noa Sher, Zhiqiang Xiong, Thomas Hankeln, Zhiyong Huang, Vera Gorbunova, Lu Zhang, Wei Zhao, Derek E. Wildman, Yingqi Xiong, Andrei Gudkov, Qiumei Zheng, Gideon Rechavi, Sanyang Liu, Lily Bazak, Jie Chen, Binyamin A. Knisbacher, Yao Lu, Imad Shams, Krzysztof Gajda, Marta Farré, Jaebum Kim, Harris A. Lewin, Jian Ma, Mark Band, Anne Bicker, Angela Kranz, Tobias Mattheus, Hanno Schmidt, Andrei Seluanov, Jorge Azpurua, Michael R. McGowen, Eshel Ben Jacob, Kexin Li, Shaoliang Peng, Xiaoqian Zhu, Xiangke Liao, Shuaicheng Li, Anders Krogh, Xin Zhou, Leonid Brodsky \& Jun Wang

Nature Communications 5:3966 doi: 10.1038/ncomms4966 (2014); Published online 3 Jun 2014; Updated 12 Aug 2015

In Fig. 2 of this Article, the CLOCK protein sequence of the blind mole rat (Spalax) was inadvertently used to represent that of the naked mole rat (Heterocephalus glaber) in both the sequence alignment (Fig. 2a) and the resulting phylogenetic tree (Fig. 2b). On the basis of this analysis, it was proposed that the expanded glutamine-rich region of these proteins arose by convergent evolution. However, an alignment and phylogenetic tree using the correct sequences (new Fig. 2a,b) does not support this conclusion. We thank Frédéric Delsuc for identifying this error, which does not affect the main findings of the paper. 
a

Rattus
Mus
Spalax
Heterocephalus
Homo

Rattus
Mus
Spalax
Heterocephalus
Homo

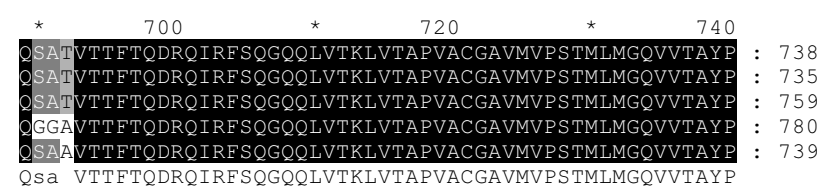

Rattus
Mus
Spalax
Heterocephalus
Homo
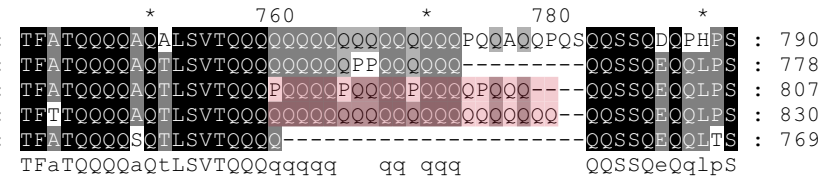

800

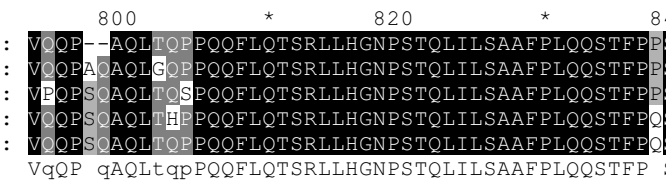

840

Homo

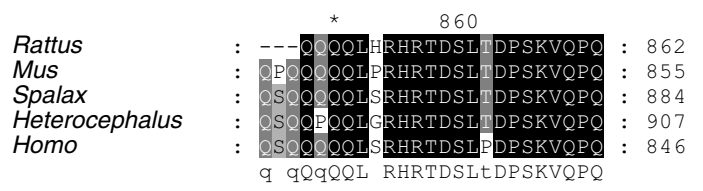

b

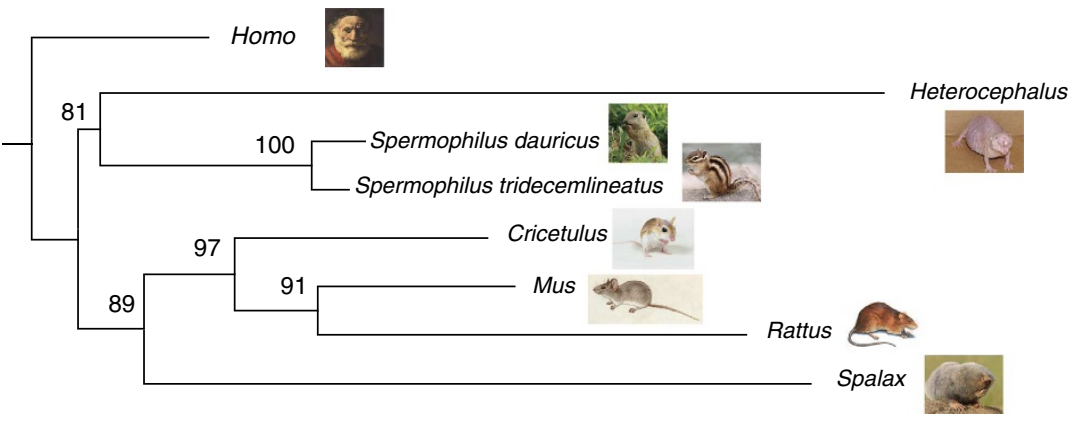

Divergence, substitutions/site

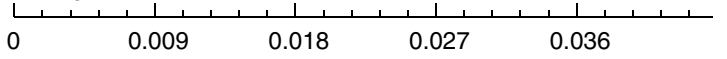

Figure 2 | Evolution of BMR and NMR CLOCK proteins. (a) The Q-rich domain of BMR (Spalax) CLOCK proteins compared with that of NMR (Heterocephalus), human (Homo), rat (Rattus) and mouse (Mus). Red box indicates the expanded glutamine-rich area in BMR with 4 P substitutions. (b) Phylogenetic tree of the CLOCK protein. The rooted tree describes the similarity relationships among the CLOCK proteins of BMR, NMR, mouse, rat, thirteen-lined ground squirrel (Spermophilus tridecemlineatus), Daurian ground squirrel (S. dauricus), Chinese hamster (Cricetulus) and human (Homo). 